\title{
Viability of Milk Neutrophils and Severity of Bovine Coliform Mastitis
}

\author{
J. Mehrzad,* L. Duchateau, and C. Burvenich \\ Ghent University, Faculty of Veterinary Medicine, Department of Physiology, \\ Biochemistry and Biometrics, Salisburylaan 133, B-9820 Merelbeke, Belgium
}

\begin{abstract}
To study the host-pathogen interactions during Escherichia coli mastitis, we first determined whether $E$. coli infection would change blood and milk polymorphonuclear neutrophil (PMN) chemiluminescence (CL) and viability. We then hypothesized that when $E$. coli invade the mammary gland, the viable PMN in milk would efficiently phagocytose and destroy $E$. coli before establishment of infection. We observed that the phagocytosis-dependent and independent CL were closely linked to PMN viability and were crucial to the outcome of mastitis. Maximal PMN influx and colony-forming units in infected quarters appeared at postinfection hours (PIH) 6 to 24. This further boosted PMN recruitment through bone marrow-blood barrier as well as blood-milk barrier. The survival of recruited PMN in the $E$. coli-infected quarters was much higher than that of noninfected quarters. Chemiluminescence activity of PMN from the infected quarters significantly increased following $E$. coli infection, even exceeding that of blood at PIH 6, 12, and 18 to 24; no such increase was observed in noninfected quarters, suggesting that the various responses of milk PMN to stimuli resulted largely from PMN viability. The highest CL intensity and durability was observed in milk PMN from infected quarters at PIH 12. Whereas an increased viability of PMN in the noninfected quarters was only significant at PIH 6 , the viability of PMN in infected quarters was long lasting and significantly higher at PIH 6 to 72 . Importantly, higher preinfection milk PMN viability correlated with bacterial clearance, which was accompanied by faster recovery. Our study strongly supports the hypothesis that boosting milk PMN viability could be a strategy with which to prevent or reduce the severity of coliform mastitis in dairy cows. This strategy might be achieved through strengthening bone marrow functionality.
\end{abstract}

\footnotetext{
Received February 2, 2004.

Accepted August 9, 2004.

Corresponding author: C. Burvenich; e-mail: christian.burvenich @ugent.be.

*Current address: McGill University, Department of Animal Science, 111 Lakeshore Road, Ste. Anne de Bellevue, Quebec, Canada, H9X 3V9.
}

(Key words: Escherichia coli mastitis, neutrophil, severity, viability)

\begin{abstract}
Abbreviation key: AUC = area under the curve, $\mathbf{C L}=$ chemiluminescence, $\mathbf{M P}=$ daily milk production, $\mathbf{P I H}=$ postinfection hour, PMA = phorbol 12-myristate 13acetate, $\mathbf{P M N}$ = polymorphonuclear neutrophil, ROS = reactive oxygen species.
\end{abstract}

\section{INTRODUCTION}

Few early lactation-related infections in dairy cows are as critical as mastitis; even fewer have been so broadly troubling in bovine and dairy industries. Acute Escherichia coli mastitis in early-lactating cows is accompanied by severe clinical symptoms (Hill et al., 1979; Vandeputte-Van Messom et al., 1993). Polymorphonuclear neutrophils (PMN) are pivotal in protecting the cow's udder from $E$. coli infection (Kehrli and Shuster, 1994; Paape et al., 2002). Intramammary innate defense against invading pathogens relies heavily on the number of circulating PMN before infection and their capacity to produce reactive oxygen species (ROS; Heyneman et al., 1990). It also depends on the rate of PMN diapedesis into the infected quarters (Hill et al., 1979; Vandeputte-Van Messom et al., 1993), and on the activity of bone marrow to provide young/active PMN (Van Merris et al., 2002; Burvenich et al., 2003). Furthermore, PMN viability, which evidently reflects the pathophysiological condition of the mammary gland (Piccinini et al., 1999; Mehrzad et al., 2001a, b), could play a role in the innate defense mechanism.

Polymorphonuclear neutrophil ROS quantification after PMN stimulation with latex or with phorbol 12myristate 13-acetate (PMA) using a chemiluminescence $(\mathbf{C L})$ assay is a reproducible technique to investigate the phagocytosis or bactericidal system of PMN (Allen et al., 1972; Grebner et al., 1977; Piccinini et al., 1999; Mehrzad et al., 2001c). In nonmastitis cows, rarely has the milk PMN viability vs. CL been investigated. The milk viability of PMN, which represents their quality, could reflect the dynamic of PMN recruitment not only through bone marrow-blood barrier but also through blood-milk barrier. Throughout the lacta- 
tion cycle, milk PMN CL in healthy cows varies from 47 to $78 \%$ of their blood counterparts (Mehrzad et al., 2001b); similar variation might exist for milk PMN viability. These variations can be due to the impact of parturition and lactation alone (Mehrzad et al., 2001b; Van Oostveldt et al., 2002). Clearly, blood PMN recruit into $E$. coli-infected and noninfected quarters, and assessing the functionality of the PMN in both quarters might shed some light on the complex pathophysiology of $E$. coli mastitis. The fluctuations between milk and blood PMN CL and viability during endotoxin mastitis have been shown to be different from those caused by physiological conditions of the cow and the udder (Mehrzad et al., 2001a; b). It is debatable whether these results can be extrapolated to coliform mastitis. Thus, comparison of milk PMN functions after normal diapedesis (before infection or during infection in noninfected quarters) and accelerated diapedesis (in E. coli-infected quarters) with those of blood offers additional details about host-pathogen interactions during $E$. coli mastitis. We speculate that the impact of fast PMN diapedesis (rapidly increased SCC on PMN quality and their ROS production capability) could cause dissimilarities between milk PMN from infected and noninfected quarters. Furthermore, the resident milk PMN viability could provide an efficient phagocytosis capacity for the mammary gland at the start of the $E$. coli infection. In addition, the relationship between postinfection bacterial growth in milk and preinfection milk PMN viability has not yet been demonstrated. The outcome of mastitis may also be linked to preinfection milk PMN viability.

The phagocytosis process of $E$. coli in milk by PMN is energy-dependent and requires the presence of a functional cytoskeleton and protruding pseudopods (Paape et al., 2003). Permanent activity of NADPHoxidase (initiator of CL or ROS or both) is linked to the actin filaments of PMN (Sandgren et al., 1992), and CL impairment causes fast PMN lysis/necrosis during $E$. coli phagocytosis (Goldberg et al., 1995), boosting phagocytosis inefficiency. Therefore, internalization of bacteria by PMN is associated with activation and reorganization of the cell cytoskeleton (Meconi et al., 2001). The cytoskeleton machinery is considered to envelope the bacteria in a membrane-zippering mechanism (Griffin et al., 1975). This mechanism in the milk compartment would not occur unless the PMN are viable. Furthermore, PMN cytosolic $\mathrm{pH}$ hemostasis, as inhibitor of PMN necrosis, results, in part, from PMN ROS production (Grebner et al., 1977; Mayer et al., 1989; Jankowski et al., 2002; Reeves et al., 2002); this suggests direct or indirect influence of PMN CL on PMN viability. The concomitant assessment of PMN viability and CL in milk would provide insight into the cytochemical, cytoskeletal, phagocytosis, and bactericidal status of the PMN; this could be representative of the most complex part of PMN-E. coli interaction in the udder during phagocytosis.

To examine if there is any parallel relationship between blood and milk (infected and noninfected quarters) PMN function before, during, and after $E$. coli mastitis, the effect of $E$. coli infection on milk PMN viability and CL was assessed. The impact of preinfection milk PMN viability on bactericidal capacity in the gland, and on the milk production performance (as indicator for the severity of mastitis), was also investigated. The CL technique for ROS production assessment was used as an appropriate indicator for PMN viability fluctuations. Polymorphonuclear neutrophil maturity (as indicator for bone marrow activity) during $E$. coli mastitis was also determined to gain insight into the observed changes in PMN CL and viability. The kinetics of blood and milk PMN CL during peak PMN functional and structural changes was studied in detail.

\section{MATERIALS AND METHODS}

This experiment has been approved by the ethical committee of the Faculty of Veterinary Medicine of Ghent University.

\section{Animals and Experimental Procedures}

For the E. coli trial, 35 Holstein-Friesian Red pied breed cows in their $224 \pm 15 \mathrm{~d}$ of first pregnancy (2.4 \pm $0.3 \mathrm{yr}$ ) on arrival at the experimental dairy farm were studied. The animals, on a zero-grazing system from arrival until the end of the experiment, were put in individual stalls and were fed with a special ration for pregnancy and lactation. They had free access to water and hay. After gestation, clinically healthy cows, showing no sign of typical periparturient diseases, were eventually selected based on 2 consecutive bacteriological negative milk samples and a milk SCC of $<2 \times 10^{5}$ / $\mathrm{mL}$ of milk per individual quarter. At $1 \mathrm{wk}$ before the start of the experiment, the animals were fed a daily ration of approximately $8 \mathrm{~kg}$ of concentrate and had free access to water and hay. They were milked twice daily at 0800 and $1700 \mathrm{~h}$ with a 4-quarters milking machine. Escherichia coli were inoculated into the udder of animals at $20 \pm 5 \mathrm{~d}$ after parturition.

In the first study, 15 cows were selected. Individual quarter milk samples were aseptically collected for determination of colony-forming units $(10 \mathrm{~mL}), \mathrm{SCC}(50$ $\mathrm{mL}$ ), and isolation $(200 \mathrm{~mL})$ at $24 \mathrm{~h}$ before, immediately before, and at $6,12,18,24,48,72,144,216$, and 312 $\mathrm{h}$ following $E$. coli infection. For bacteriological examination of milk, $0.5 \mathrm{~mL}$ of quarter milk was serially diluted in a pyrogen-free saline solution $(0.9 \%)$, and 
$0.01 \mathrm{~mL}$ of the diluted sample was streaked in duplicate on Columbia sheep blood agar (Biokar Diagnostics, Beauvois, France) plates, using an inoculation loop. The plates were incubated for $24 \mathrm{~h}$ at $37^{\circ} \mathrm{C}$ for enumeration (cfu) of the milk samples. Peripheral blood $(80 \mathrm{~mL})$ was collected aseptically from each cow by venipuncture from the external jugular vein into evacuated tubes (Laboratory EGA, F-28210 Nogent le Roi, France) containing $125 \mathrm{IU}$ of heparin as anticoagulant. Subsequent blood sampling was carried out after milk sampling at $24 \mathrm{~h}$ before, immediately before, and at $6,12,18,24$, $48,72,144,216$, and $312 \mathrm{~h}$ after $E$. coli infection. Measurements of rectal temperature, heart rate, rumen motility, and clinical examination of the mammary gland were performed at the time of blood and milk sampling. Evening and morning milk was pooled to obtain daily milk production (MP).

In the second study, 20 cows were investigated for experimentally induced $E$. coli mastitis to assess the impact of preinfection milk PMN viability on the $E$. coli growth in the mammary gland at postinfection $\mathrm{h}(\mathbf{P I H})$ 6 and on the MP loss at PIH 48 (a strong indicator of the severity of mastitis).

In a third study, to investigate the eventual relationship between milk PMN viability and CL, 66 clinically healthy Holstein-Friesian cows from the Ghent University dairy farm (Biocentrum Agri-Vet Melle, Belgium), in their first to fourth parity, early- $(\mathrm{n}=33,21 \pm 6 \mathrm{~d}$ of lactation), and mid-lactating cows ( $\mathrm{n}=33,210 \pm 36$ $\mathrm{d}$ of lactation) were selected for milk sampling. The cisternal quarter milk samples $(500 \mathrm{~mL})$ were aseptically collected, using a sterile teat cannula. The milk PMN viability values were simultaneously compared with their CL values.

\section{Bacterial Challenge}

Escherichia coli $\mathrm{P} 4: 032$ isolated from a clinical case of mastitis was used. The stock of $E$. coli was maintained in lyophilized medium at $-20^{\circ} \mathrm{C}$ until use and frequently controlled for viability and purity. To prepare the inoculum, the bacteria were subcultured in brain heart infusion broth (CM225; Oxoid, Nepean, ON) at $37^{\circ} \mathrm{C}$. The bacterial suspensions were washed 3 times with pyrogen-free saline solution $(0.9 \%)$ and resuspended in the solution. Bacterial counting was performed using the plate count method to obtain the desired concentration. Before $E$. coli infection, the teat ends were disinfected with $70 \%$ ethanol mixed with $0.5 \%$ chlorohexidine. At 20 min after the morning milking, $E$. coli mastitis was induced into the left udder half by a single intramammary inoculation of $10 \mathrm{~mL}$ of $10^{4}$ cfu of $E$. coli P4:032 solution per quarter using a sterile teat cannula (7 cm; Me. Ve. Mat., Deinze, Belgium).
After inoculation, each quarter was gently massaged for $30 \mathrm{~s}$ to distribute the bacterial solution in the gland.

\section{Blood and Milk PMN Preparation and Enumeration}

All materials and reagents used for the isolation of blood and milk PMN were sterile. Isolation of PMN from peripheral blood was performed using hypotonic lysis of erythrocytes. Briefly, $40 \mathrm{~mL}$ of heparinized blood was poured into the Falcon tubes and centrifuged (1000 $\times g, 15 \mathrm{~min}, 4^{\circ} \mathrm{C}$ ); the plasma layer, buffy coat, and top layer of the blood-packed cells were discarded. About $10 \mathrm{~mL}$ of the blood-packed cell was lysed by adding 20 $\mathrm{mL}$ of double distilled water and gently mixed for $45 \mathrm{~s}$ using a magnetic stirrer. After restoration of the isotonicity by addition of $10 \mathrm{~mL}$ of $2.7 \% \mathrm{NaCl}$ with gentle mixing for $60 \mathrm{~s}$, the suspension was centrifuged (1000 $\times g, 10 \mathrm{~min}, 4^{\circ} \mathrm{C}$ ). For the second lysis procedure, after resuspending of the pellets in $10 \mathrm{~mL}$ of Dulbecco's PBS (Gibco BRL, Life Technologies Inc., Gaithersburg, MD), $20 \mathrm{~mL}$ of double distilled water was added and gently mixed for $30 \mathrm{~s}$, then $10 \mathrm{~mL}$ of $2.7 \% \mathrm{NaCl}$ was added, gently mixed for $60 \mathrm{~s}$, and centrifuged $(1000 \times g, 5 \mathrm{~min}$, $4^{\circ} \mathrm{C}$ ). The remaining cell pellet was washed 3 times in PBS $\left(300 \times g, 10 \mathrm{~min}, 4^{\circ} \mathrm{C}\right)$ and the final cell pellet was resuspended in 1 to $2 \mathrm{~mL}$ of PBS with gelatin $(0.5 \mathrm{mg} /$ $\mathrm{mL}$; Merck, Darmstadt, Germany) for further analyses. The isolation procedure of PMN from blood yielded $>98 \%$ of granulocytes (PMN + eosinophils) with predominantly PMN ( $>85 \%)$. After counting the cells using an electronic particle counter (Coulter counter Z2, Coulter Electronics Ltd., Luton, UK) and determining the viability (see subsequently) and percentage of PMN, the cell suspensions were adjusted to a concentration of 5 $\times 10^{6}$ cells $/ \mathrm{mL}$ in PBS supplemented with gelatin $(0.5$ $\mathrm{mg} / \mathrm{mL}$ ).

In the first study, individual quarter milk samples were used for subsequent PMN isolation as described previously (Mehrzad et al., 2001a). Briefly, pooled milk of the $2 E$. coli-infected and the 2 noninfected quarters of each cow was filtered separately through a nylon filter (40 $\mu \mathrm{m}$ pore size) and diluted to $60 \%$ with cold PBS (vol/vol). In the second study, only infected quarter milk samples were used for PMN preparation and enumeration. Isolation of PMN from milk was performed using 3 centrifugation steps as previously described (Mehrzad et al., 2001a, b). The total number of leukocytes and isolated blood and milk cells were determined using an electronic particle counter (Mehrzad et al., 2001b). The total number of different circulating leukocytes was determined using smear preparations of blood samples (Mehrzad et al., 2001b). Differential cell counts and staining procedures were performed on whole blood similar to the isolates on eosin-Giemsa- 
stained smears, using light microscopy. Cell identification was based on morphological characteristics as described by Mehrzad et al., (2001b). To quantify percentages of each cell type in the samples, 200 cells per slide were classified as PMN (mature and immature), monocytes/macrophages, lymphocytes, eosinophils, or epithelial cells (only in milk).

\section{Viability of Milk and Blood PMN}

The viability of isolated PMN from blood and milk (infected and noninfected) quarters was determined in duplicate by means of flow cytometry (FACScan, Becton Dickinson Immunocytometry Systems, San Jose, CA), using propidium iodide exclusion (Mehrzad et al., 2001b). The percentage of propidium iodide-positive PMN was calculated to quantify necrotic PMN for the later calculation of percentage of viable PMN.

In the second study, milk PMN viability of infected quarters, before and after infection, was assessed. The viability of PMN isolated from blood and milk of $E$. coliinfected and noninfected quarters were measured at PIH 6, 12, 18, 24, 48, 72, 144, 216, and 312.

\section{ROS Production of Milk and Blood PMN}

Polymorphonuclear neutrophil ROS production was quantified using CL assay; luminol-amplified cellular CL, stimulated with PMA and latex beads (polystyrene, $0.76 \mu \mathrm{m}$ diameter, $4 \times 10^{11} \mathrm{particles} / \mathrm{mL}$; Sigma), was applied for CL of PMN isolated from blood and milk of E. coli-infected and noninfected quarters. Chemiluminescence was measured in duplicate during $30 \mathrm{~min}$ at $37^{\circ} \mathrm{C}$ with a microtiter plate luminometer (type LB96P; EG\&G Berthold, Bad Wildbad, Germany). Phorbolstimulated CL was measured immediately after addition of $100 \mathrm{ng} / \mathrm{mL}$ of PMA and $0.3 \mathrm{mM}$ luminol (5-amino2, 3-dihydro-1, 4-phthalazinedione; Sigma) to $2 \times 10^{6}$ cells $/ \mathrm{mL}$ in a total volume of $200 \mu \mathrm{L}$ per well. Similar concentrations of luminol and cells per well were used for CL stimulated with latex beads (final concentration of 500 particles/PMN). Stock solutions of PMA and luminol were prepared in dimethyl sulfoxide (Sigma) and stored at $-20^{\circ} \mathrm{C}$. The area under the curve (AUC) was calculated for the registered impulse rates over the entire measurement period of $30 \mathrm{~min}$. The CL response was expressed per $10^{3}$ viable PMN in each isolated cell sample. Because the contribution of milk macrophages to luminol-dependent CL is negligible (Mehrzad et al., $2001 b$ ), the CL response was expressed per $10^{3}$ viable PMN and obtained as follows:

$$
C L_{P M N}=\frac{10^{3} \times A U C}{N_{C E L L S} \times \% P M N \times \% V I A B}
$$

where AUC = the area under the curve during the period of 30 min of CL measurement, $\mathrm{N}_{\mathrm{CELLS}}=$ the number of cells in the sample, equal to $4 \times 10^{5}, \% \mathrm{PMN}=$ the percentage of PMN in the sample, and \%VIAB = the percentage viable PMN in the sample.

The CL of blood PMN was calculated with the same formula as for milk PMN applying the corrections described by Heyneman et al. (1990) and Mehrzad et al. (2001a) to correct for interference of eosinophils. Chemiluminescence of PMN isolated from blood and milk of E. coli-infected and noninfected quarters was measured at PIH 6, 12, 18, 24, 48, 72, 144, 216, and 312.

The CL kinetics of PMA- and latex-stimulated and nonstimulated and/or resting blood and milk ( $E$. coli infected and noninfected quarters) PMN were evaluated in detail at PIH $0,12,24$, and 72 .

\section{Relation Between PMN Viability and CL}

In the third study, to assess the correlation between PMN viability and latex-stimulated CL, nonmastitic cows were further tested to determine whether there was any connection between milk PMN CL and viability.

\section{Statistical Analyses}

A mixed model was fitted to the CL data (AUC of 1000 viable PMN/30 min), including cow as random effect and period (as a categorical variable with levels PIH 0, 6, 12, 18 to 24,48 to 72 , and $>72$ ), PMN stimulation methods (latex, PMA, or nonstimulated) and location of PMN (blood, E. coli-infected quarters, and noninfected quarters) and their 2-way interactions as fixed effects. To study the time evolution of CL after infection, CL in each period was compared with CL just before the infection (time 0) in each of the different settings (method by location combinations) at a Bonferroni multiple comparisons adjusted significance level of 0.01 (5 comparisons). A similar mixed model (but without stimulation method) was fitted to the viability data, and the time evolution was analyzed in detail as in the previous analysis.

In the second study, the effect of preinfection milk PMN viability on E. coli growth at PIH 6 and MP loss at PIH 48 was analyzed using regression analysis, to determine if the slope expressing the linear effect of preinfection milk PMN viability was significantly different from zero. In the third study, the Spearman correlation coefficient was used to assess the correlation between milk PMN viability and CL. It was further determined if this correlation coefficient was significantly different from zero. 
Table 1. Latex-, $\mathrm{PMA}^{1}$-, and nonstimulated chemiluminescence of blood and milk (infected and noninfected quarters) polymorphonuclear neutrophils (PMN) before and during experimentally induced Escherichia coli mastitis. Values are means \pm SEM of 15 cows. Data are expressed as the area under the curve of continuously reactive oxygen species generation of $10^{3}$ viable PMN for $30 \mathrm{~min}$. Each timepoint is compared with postinfection hour (PIH) 0 (before challenge), with the asterisks denoting a significant difference at comparisonwise error rate equal to 0.01 .

\begin{tabular}{lllrl}
\hline & & \multicolumn{3}{c}{ Source of PMN } \\
\cline { 3 - 5 } Stimulator & & \multicolumn{1}{c}{$\begin{array}{c}\text { Infected } \\
\text { quarters }\end{array}$} & $\begin{array}{l}\text { Noninfected } \\
\text { quarters }\end{array}$ \\
\hline PMA & PIH & $3925 \pm 510$ & $1086 \pm 512$ & $1044 \pm 513$ \\
& 0 & $3763 \pm 696$ & $7710 \pm 691^{* *}$ & $1646 \pm 693$ \\
& 6 & $2739 \pm 691$ & $11395 \pm 697^{* *}$ & $1583 \pm 685$ \\
& 12 & $3150 \pm 514$ & $10237 \pm 512^{* *}$ & $1634 \pm 512$ \\
& 18 to 24 & $7555 \pm 514^{* *}$ & $3003 \pm 512^{* *}$ & $1553 \pm 513$ \\
Latex & 48 to 72 & $4245 \pm 436$ & $1800 \pm 431$ & $1261 \pm 430$ \\
& $>72$ & $3153 \pm 514$ & $881 \pm 510$ & $950 \pm 513$ \\
& 0 & $2684 \pm 698$ & $6017 \pm 697^{* *}$ & $1484 \pm 695$ \\
& 6 & $2480 \pm 694$ & $8697 \pm 691^{* *}$ & $1942 \pm 692$ \\
& 12 & $4701 \pm 514$ & $8076 \pm 512^{* *}$ & $1477 \pm 513$ \\
Nonstimulated & 18 to 24 & $2767 \pm 514^{* *}$ & $1540 \pm 512^{* *}$ & $1108 \pm 510$ \\
& 48 to 72 & $6955 \pm 436$ & $686 \pm 512$ & $1165 \pm 431$ \\
& $>72$ & $722 \pm 697$ & $2778 \pm 695$ & $844 \pm 513$ \\
& 0 & $895 \pm 524$ & $4571 \pm 697^{* *}$ & $1246 \pm 692$ \\
& 6 & $1403 \pm 511$ & $3535 \pm 514^{* *}$ & $1835 \pm 690$ \\
& 12 & $2255 \pm 509$ & $1343 \pm 512$ & $1230 \pm 512$ \\
& 18 to 24 & $916 \pm 430$ & $1237 \pm 431$ & $1083 \pm 507$ \\
& 48 to 72 & & & $1081 \pm 432$ \\
\hline
\end{tabular}

$* * P=0.01$.

${ }^{1} \mathrm{PMA}=$ Phorbol 12-myristate 13-acetate.

\section{RESULTS}

\section{ROS Production of Milk and Blood PMN}

During $E$. coli infection, milk PMN CL increased far more rapidly than CL in blood and noninfected quarters PMN (Table 1 and Figure 1a, b, c). Compared with preinfection values, ROS load of PMA-stimulated milk PMN from $E$. coli-infected quarters significantly increased 7.1-, 10.5-, 9.4-, and 2.8-fold at PIH 6, 12, 18 to 24 , and 48 to 72 , respectively. The ROS load for latex stimulation significantly increased 6.8-, 9.8-, 9.1-, and 3 -fold at the same timepoints, respectively (Table 1; Figure 1b). At PIH 6 to 72, only a slight nonsignificant increase of CL was observed in noninfected quarters (Table 1; Figure 1c). For blood PMN, ROS load differed significantly from preinfection values only at PIH 48 to 72 , at which it roughly doubled (from AUC 3925 to 7555) for PMA and increased 2.8-fold (from AUC 3153 to 8767) following latex stimulation (Table 1; Figure 1a).

Nonstimulated PMN CL paralleled the PMA- and latex-stimulated PMN CL for blood and milk (infected and noninfected quarters) PMN. A significant difference with preinfection values was only observed in the infected quarters at PIH 12 and PIH 18 to 24 (Table 1; Figure 1c). The preinfection PMA-stimulated CL was higher than latex-stimulated CL in both milk and blood

Figure 2 shows typical CL profiles of PMA-stimulated, latex-stimulated, and nonstimulated blood and milk (infected and noninfected quarters) PMN of 5 infected cows at PIH 0, 12, 24, and 72. The shape differed, somehow, for PMA- and latex-stimulated PMN CL, especially for PMN from $E$. coli-infected quarters. The CL response for $E$. coli-infected quarters PMN following PMA stimulation showed a biphasic course, with an immediate large peak followed by a delayed small peak at PIH 12 and 24. During the same period, maximum CL of milk PMN from noninfected quarters increased slightly. At PIH 72, CL intensity and durability did not alter significantly in PMN of either quarter.

\section{Viability of Milk and Blood PMN}

The viability of blood PMN did not change significantly throughout the experiment and constantly remained high $(98 \pm 1 \%)$. Before $E$. coli challenge, the viability of PMN isolated from milk was $63 \pm 2 \%$. The viability of PMN isolated from milk of $E$. coli-infected quarters at PIH 6, 12, 18 to 24 , and 48 to 72 increased to $86,93,89$, and $76 \%$, respectively $(P<0.01)$. Preinfection values were obtained at PIH $>72$ (Table 2 ). The viability of PMN isolated from milk of noninfected quarters at PIH 6 increased significantly to $77 \%(P<0.01)$ (Table 2$)$.

\section{Preinfection Milk PMN Viability vs. Bacterial Growth in Milk and MP Loss}

In the second study, we evaluated the link between residual milk PMN viability and the inhibition of bacte- 


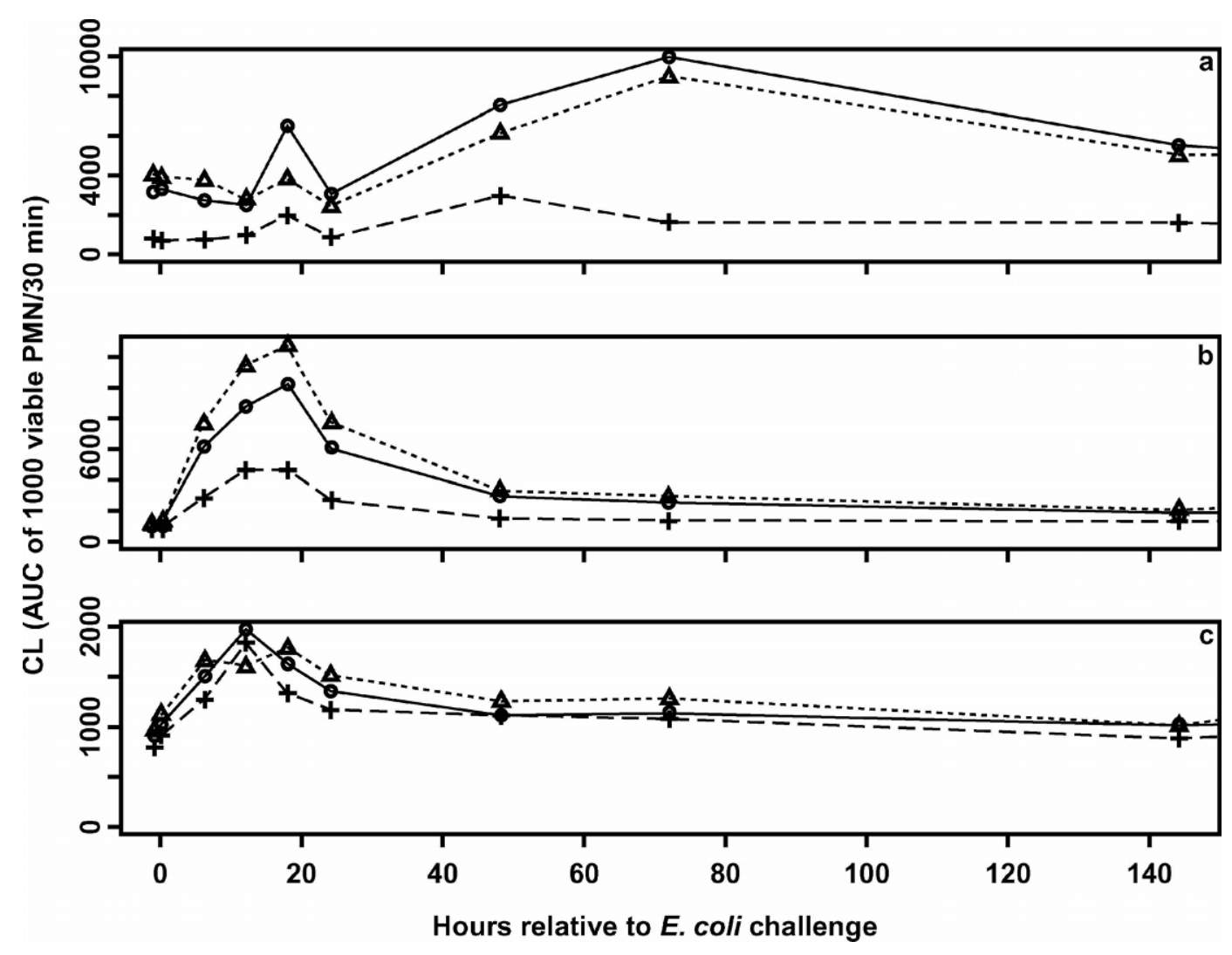

Figure 1. Phorbol 12-myristate 13-acetate (PMA)-stimulated (dotted lines), latex-stimulated (solid lines), and nonstimulated (dashed lines) chemiluminescence (CL) of polymorphonuclear neutrophils (PMN) isolated from blood (a), E. coli-infected quarter (b), and noninfected quarter (c) of cows during experimentally induced $E$. coli mastitis. Values are the mean of 15 cows. Data are expressed as the area under the curve (AUC) of continuously reactive oxygen species (ROS) generation of $10^{3}$ viable PMN for 30 min.

rial growth in the milk after $E$. coli infection. There was a strong negative correlation between preinfection milk PMN viability and bacterial growth at PIH $6(P<$ 0.01 ; Figure 3a). There was also an inverse relationship between preinfection milk PMN viability and MP loss at PIH $48(P<0.05$; Figure $3 \mathrm{~b})$.

\section{Relation Between PMN Viability and CL}

In the third study, there was a significant positive correlation between milk PMN viability and latex-stimulated CL ( $\rho=0.94 ; P=0.0001$; Figure 4 ). The 2 parameters, viability and CL, in milk were inextricably interrelated. During early lactation, the milk PMN CL was lower, and PMN survival in milk was also lower; however, these values were significantly high during midlactation.

\section{Clinical Observations, Enumeration, and Differentiation of Blood and Milk PMN}

Acute mastitis caused by $E$. coli inoculation provoked local as well as systemic effects: inflammation of the
$E$. coli-infected quarters with an increase of colonyforming units and SCC, decreased MP, and increased rectal temperature, pulse, and respiration. Most clinical signs peaked at PIH 6 to 12 and completely restored at PIH 72 to 96 (data not shown).

Leukopenia was observed between PIH 12 and 18, returning to around preinfection values at PIH 48 and onwards (Table 3). The number of circulating PMN decreased by one-third at PIH 6 , reached minimal values at PIH 12, and remained remarkably low at PIH 18 to 24, 48 to 72 , and $>72$ (Table 3 ). The number of band cells in the circulation doubled at PIH 6, was slightly lower at PIH 12, roughly tripled at PIH 18 to 72 , and remained substantially high at PIH $>72$. Metamyelocytes and myelocytes were increased at PIH 6, peaking at PIH 48 to 72 , and remained high at PIH $>72$ (Table 3 ).

At PIH 6 to 24, a sharp increase of SCC was observed in $E$. coli-infected quarters, coinciding with sharply increased colony-forming units at PIH 6 . There was an inverse relationship between SCC and colony-forming units at PIH 12, 18, and 24: the level of colony-forming units declined substantially, but was still high, at these 


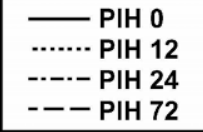

Blood
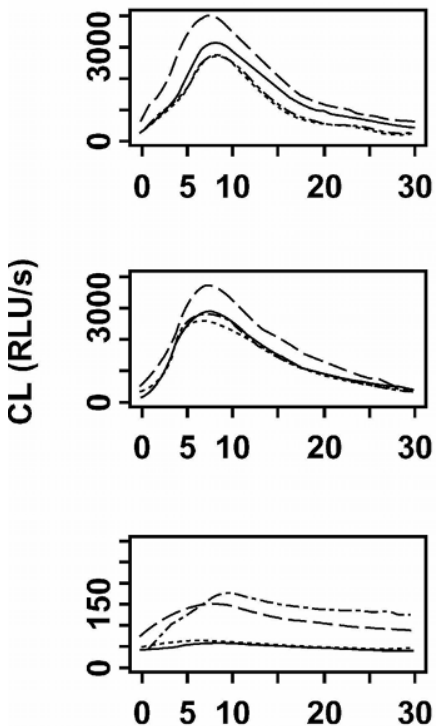

Infected quarters
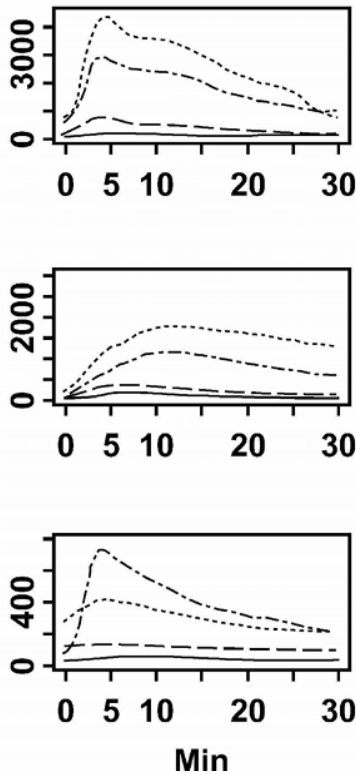

Noninfected quarters
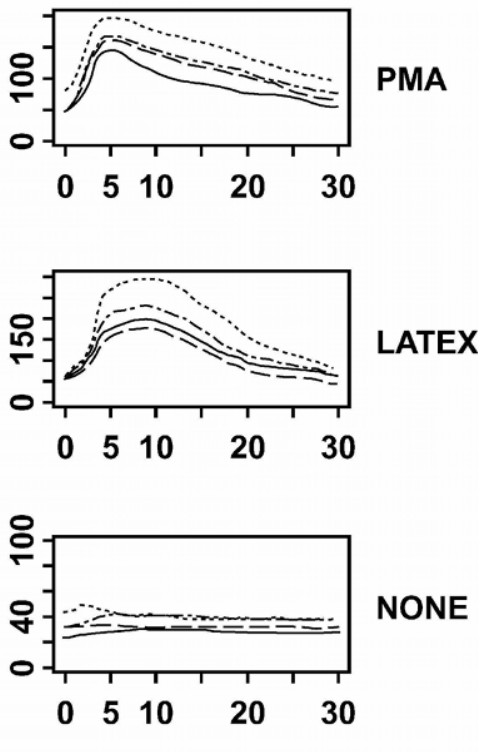

NONE

Figure 2. Chemiluminescence (CL) profile of phorbol 12-myristate 13-acetate (PMA)-stimulated (upper row), latex-stimulated (middle row), and nonstimulated (bottom row) polymorphonuclear neutrophils (PMN) from blood and milk, infected and noninfected quarters, at postinfection hour (PIH) $0,12,24$, and 72 of experimentally induced $E$. coli mastitis. The generation of CL was monitored continuously for $30 \mathrm{~min}$ after addition of $100 \mathrm{ng} / \mathrm{mL}$ of PMA or 500 latex bead particles/PMN or neither followed by $0.3 \mathrm{mM}$ luminol to the $4.10^{5}$ isolated PMN suspension in $200 \mu \mathrm{L}$; CL was expressed as cumulative relative light unit (RLU)/s. The curves are average of 5 cows.

PIH (Figure 5). The SCC in control quarters did not change. Before $E$. coli infection, the percentage of PMN in isolated milk cells was $46 \pm 3 \%$. Compared with preinfection values, the percentage of milk PMN almost doubled at PIH 6 to 24 in $E$. coli-infected quarters, re-

Table 2. Viability fluctuations of blood and milk (infected and noninfected quarters) polymorphonuclear neutrophils (PMN) during experimentally induced Escherichia coli mastitis. ${ }^{1}$ Each timepoint is compared with postinfection hour (PIH) 0 (before challenge), with the asterisks denoting a significant difference at comparisonwise error rate equal to 0.01 .

\begin{tabular}{llll}
\hline & \multicolumn{3}{c}{ Source of PMN } \\
\cline { 2 - 4 } PIH & Blood & $\begin{array}{l}\text { Infected } \\
\text { quarters }\end{array}$ & $\begin{array}{l}\text { Noninfected } \\
\text { quarters }\end{array}$ \\
\hline 0 & $98.2 \pm 3.3$ & $61.7 \pm 3.8$ & $63.5 \pm 3.4$ \\
6 & $98.7 \pm 3.2$ & $85.5 \pm 3.7^{* *}$ & $77.0 \pm 3.2^{* *}$ \\
12 & $98.0 \pm 3.2$ & $92.8 \pm 3.8^{* *}$ & $66.8 \pm 3.3$ \\
18 to 24 & $98.4 \pm 2.5$ & $88.7 \pm 2.8^{* *}$ & $70.9 \pm 2.4$ \\
48 to 72 & $98.0 \pm 2.5$ & $75.3 \pm 2.7^{* *}$ & $63.6 \pm 2.5$ \\
$>72$ & $98.4 \pm 2.1$ & $62.4 \pm 2.2$ & $59.7 \pm 2.4$ \\
\hline
\end{tabular}

$* * P=0.01$

${ }^{1}$ Values are means \pm SEM of 15 cows. Data are expressed as percentages. mained a third higher at PIH 48 to 72 , and finally regained the preinfection value at $\mathrm{PIH}>72$. However, in noninfected quarters the percentage of PMN did not change during infection (data not shown).

Numerous images of younger and immature neutrophils with some morphological and functional changes were observed in milk cell smears (Figure 6). The first appearance of $E$. coli in the milk was seen at PIH 1; later visible were phagocytosed $E$. coli at PIH 3, disappearance of $E$. coli at d 1 of infection, and maximal appearance of macrophages at PIH 48 and 72 .

\section{DISCUSSION}

The inflammatory reaction in the mammary gland is accompanied by a fast influx of PMN into the milk and by a subsequent opsonin-mediated phagocytosis (Shuster et al., 1993; Kehrli and Shuster, 1994). This is the most effective mechanism against invading pathogens (Burvenich et al., 2003; Paape et al., 2003), which represents the dynamic (inflammatory) phase of the innate defense. To what extent the resident (preexisting) PMN, which constitute the static part of the 

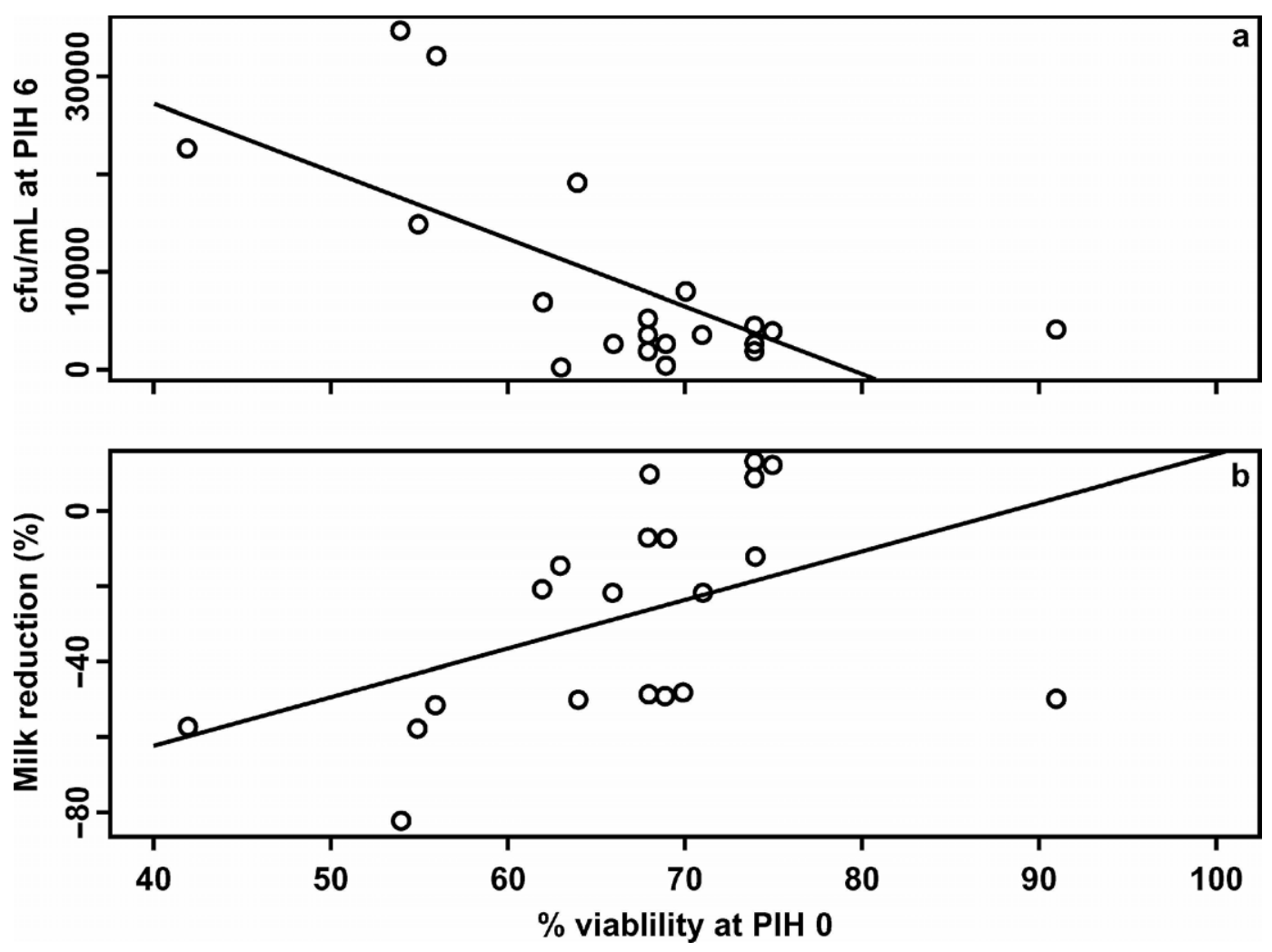

Figure 3. Correlation between preinfection milk polymorphonuclear neutrophils (PMN) viability and concentrations of bacteria in the milk at postinfection hour (PIH) 6 (a), and between preinfection milk PMN viability and milk production loss at PIH 48 (b). There is a negative correlation between preinfection milk PMN viability and bacterial growth at PIH 6 and milk production loss at PIH $48(P=0.0012$ and $P=0.041$, respectively; $\mathrm{n}=20$ ). This indicates the milk PMN effectiveness towards killing of $E$. coli and the outcome of mastitis.

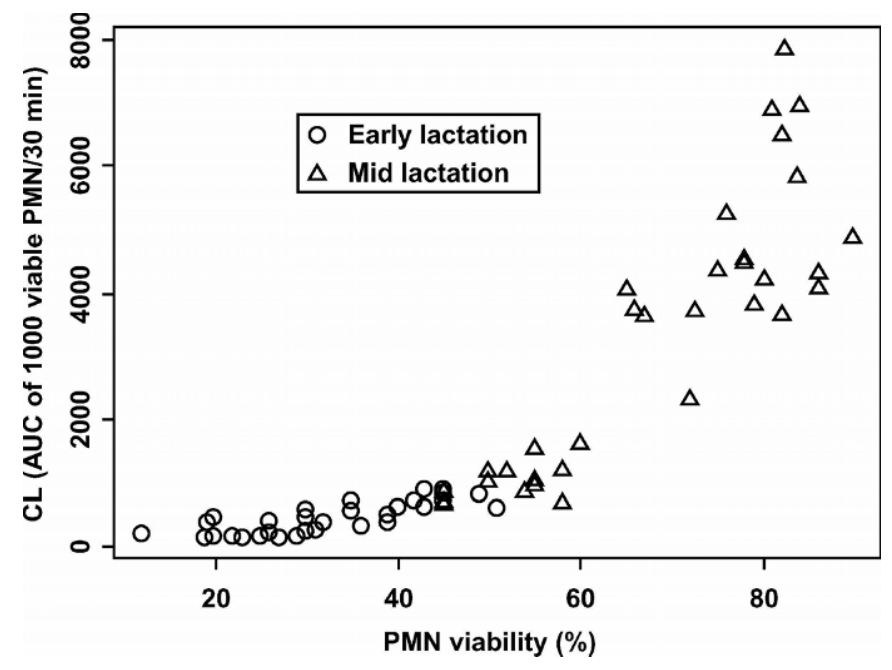

Figure 4. The Spearman correlation coefficient between milk polymorphonuclear neutrophils (PMN) latex-stimulated chemiluminescence (CL) and viability $(\mathrm{n}=66)$. There was a significant correlation between the PMN CL and their viability $(\rho=0.94 ; P=0.0001 ; \mathrm{n}=$ 66 ) during physiological conditions. The low values of CL and viability were fully related to early lactating $(\bigcirc)$ cows, $n=33,21 \pm 6 \mathrm{~d}$ of lactation, compared to mid lactating $(\triangle)$ cows, $n=33,210 \pm 36 \mathrm{~d}$ of lactation. innate defense, contribute to the udder's immunity remains unknown. The resident PMN population is apparently considered unimportant because of its low number and functionality in the absence of opsonins and inflammatory primers. Nevertheless, the direct action of the resident PMN on invading pathogens might be critical (Burvenich et al., 2003). The current study demonstrates for the first time that resident PMN in milk with low SCC may modulate the initial steps of dynamic immune defense of the udder. The study also indicates that milk PMN viability is crucially involved in the pathogenesis and outcome of coliform mastitis. Evidence in dairy cows revealed that the incidence and severity of $E$. coli mastitis were maximal during early lactation (Burvenich et al., 2003). One of the underlying causes of this could be milk PMN viability impairment (Mehrzad et al., 2001b). It is therefore reasonable to focus on and elucidate the role of milk PMN viability in the severity of coliform mastitis.

The transition from normal milk SCC to extremely high values, at which immature and mature neutrophils are released from bone marrow to the blood circulation to replenish the circulating pool (see Tables 2 and 3, Figure 5), is a stressful experience for the cow's 
Table 3. Parameters of blood cells measured during experimentally induced Escherichia coli mastitis in dairy heifers. Values are means \pm SEM of 15 cows.

\begin{tabular}{lcclcccc}
\hline & \multicolumn{5}{c}{ Parameters $^{1}$} \\
\cline { 2 - 8 } PIH $^{2}$ & WBC$/ \mu \mathrm{L}$ & PMN $/ \mu \mathrm{L}$ & Band cell $/ \mu \mathrm{L}$ & Meta + Myelo/ $\mu \mathrm{L}$ & Lymphocyte/ $\mu \mathrm{L}$ & Eosinophil $/ \mu \mathrm{L}$ & Monocyte/ $\mu \mathrm{L}$ \\
\hline 0 & $9536 \pm 8141$ & $3122 \pm 458$ & $222 \pm 45$ & $730 \pm 162$ & $4402 \pm 339$ & $828 \pm 199$ & $194 \pm 39$ \\
6 & $8888 \pm 666$ & $2080 \pm 342$ & $403 \pm 145$ & $1527 \pm 172$ & $4370 \pm 325$ & $293 \pm 69$ & $54 \pm 17$ \\
12 & $3426 \pm 736$ & $365 \pm 86$ & $173 \pm 51$ & $813 \pm 216$ & $1883 \pm 444$ & $141 \pm 62$ & $6 \pm 5$ \\
18 to 24 & $6805 \pm 621$ & $1367 \pm 249$ & $689 \pm 117$ & $1999 \pm 247$ & $2567 \pm 249$ & $179 \pm 82$ & $21 \pm 9$ \\
48 to 72 & $9174 \pm 564$ & $2142 \pm 317$ & $535 \pm 111$ & $2411 \pm 248$ & $3165 \pm 238$ & $868 \pm 149$ \\
$>72$ & $10167 \pm 255$ & $2529 \pm 249$ & $432 \pm 54$ & $1852 \pm 155$ & $4826 \pm 224$ & $686 \pm 67$ & $180 \pm 14$ \\
\hline
\end{tabular}

${ }^{1} \mathrm{WBC}=$ White blood cells; PMN = polymorphonuclear neutrophils; Meta + Myelo $=$ Metamyelocytes and myelocytes.

${ }^{2} \mathrm{PIH}=$ Postinfection hour.

bone marrow. As observed, most of the recruited PMN in milk are relatively young. The young PMN undergo slow apoptosis processing pathways and consequently survive longer (Van Merris et al., 2002; Burvenich et al., 2003). In contrast, old milk PMN are not very efficient in their function because of their intracellular glycogen depletion (Naidu and Newbould, 1973), apoptosis (Van Oostveldt et al., 2002), and decreased ROS production (Mehrzad et al., 2001b; Burvenich et al., 2003). Because PMN viability is involved in the severity of coliform mastitis, it is logical that the next phase in milk PMN research would be its manipulation via strengthening bone marrow functionality to prevent and treat mastitis. One of the attainable strategies for this would be improvement of hormonal and metabolic disorders during early lactation. This could enhance milk PMN functionality by reducing blood concentrations of, for example, ketone bodies and NEFA, conse-

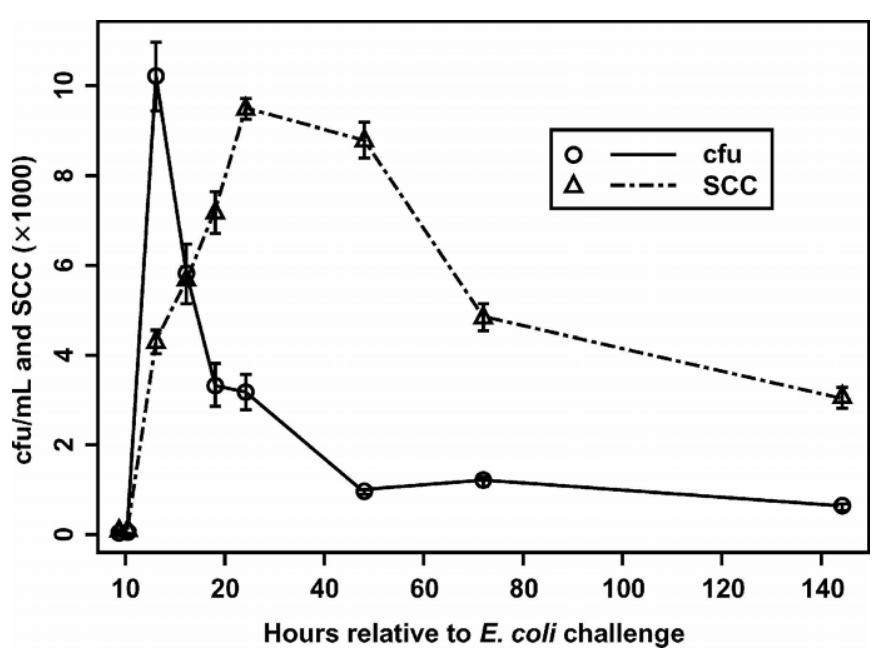

Figure 5. Multiplication rate of E. coli (solid line; $\bigcirc$ ) in infected mammary glands and pattern of leukocyte influx (dashed line; $\triangle$ ) into milk during experimentally induced $E$. coli mastitis. Values are the mean \pm SEM of 15 cows. quently modulating the proliferative capacity of myeloid cells (Hoeben et al., 1999; Burvenich et al., 2003).

Between PIH 6 and 12, a large number of PMN appeared in the lacteal secretion of inflamed quarters. This occurrence is accompanied by an increased collagenolytic and gelatinolytic activity at the level of the blood-milk barrier (Long et al., 2001; Prin-Mathieu et al., 2002), which could create a damaging effect on the barrier and lead to a partial arrest in milk secretion, potentially favoring PMN functionality in the gland (Burvenich et al., 2003). In the present study, the increased milk SCC in $E$. coli-infected quarters coincided with increased CL and viability of milk PMN, which could have been caused, in part, by the faster migration of the blood PMN. However, other phenomena may be involved, such as the release of survival factors from the inflamed area. The increase of PMN CL and viability in noninfected quarters shows that systemic factors are also involved. It is reasonable to accept that this would be the result of a faster turnover of the blood PMN due mainly to faster PMN renewal from the bone marrow.

Blood PMN CL with PMA or latex stimulation or without stimulation did not substantially change during the first day after $E$. coli infection. This is in contrast to the results of Heyneman et al. (1990), who observed a sudden decrease in PMN ROS generation. The most probable reason for this discrepancy is the use of heifers in our study. Polymorphonuclear neutrophil function in bone marrow, blood, and milk is more pronounced in heifers, compared with older cows (Mehrzad et al., 2002). The increase of latex- and PMA-stimulated blood PMN CL at PIH 48 and 72 reveals higher phagocytic and enzymatic activities. This is a rebound effect, which is typical for feedback mechanisms (Heyneman et al., 1990). In blood and noninfected quarters, PMN ROS capacity was lower with latex than with PMA. However, the opposite was true for blood and noninfected quarters at PIH 24 to 72 and 12, respectively. Conversely, in E. coli-infected quarters PMN produced more ROS with PMA than with latex. This suggests that during 

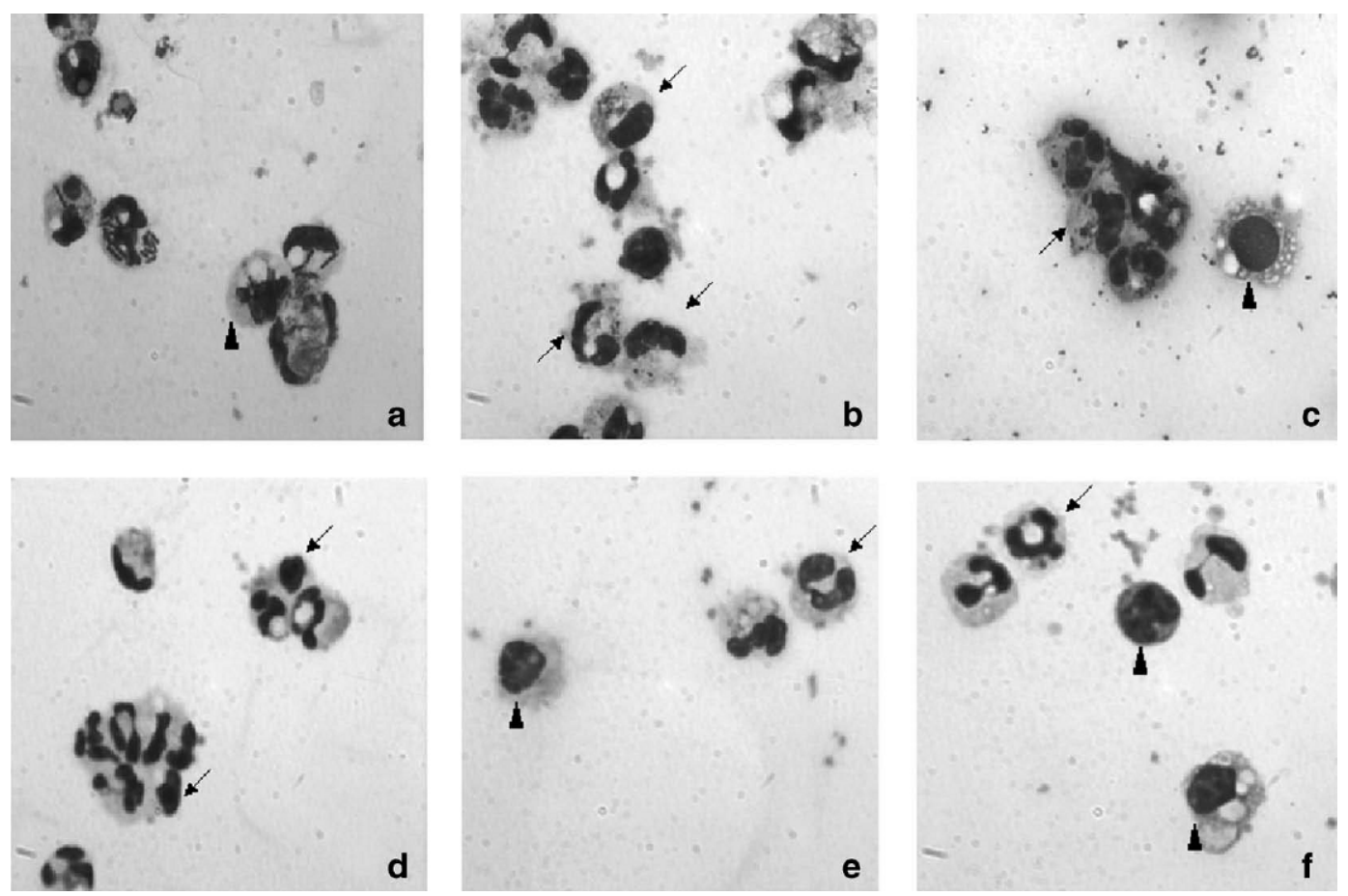

Figure 6. Light micrographs of isolated polymorphonuclear neutrophils (PMN) from $E$. coli infected quarters at postinfection hour (PIH) 6 (a), 12 (b), 18 (c), 24 (d), 48 (e), and 72 (f). E. coli is phagocytosed by milk PMN $6 \mathrm{~h}$ after the bacterial suspension was injected into the mammary gland. This representative figure shows the presence of metamyelocytes (arrows b, c, and d) with typical kidney-bean shaped nuclei, band cells (arrows e and f) with typical horseshoe-shaped nuclei, and macrophages ( $\mathbf{\Delta}$ ) with vacuolated round/spherical nuclei and whitish globules in their cytoplasm (younger macrophages had smaller whitish globules and are smaller); disappearance of $E$. coli at PIH 24, 48, and 72 is representatively shown. These morphological and functional changes boost PMN survival in milk and facilitate resolution of infection in the udder. (Hematoxylin \& Eosin staining, 1000×).

phagocytosis, ROS production is not at its maximal capacity. Evidence exists that the intracellular production of ROS is one of the most important killing mechanisms, especially for $E$. coli (Burvenich et al., 2003). Polymorphonuclear neutrophil CL of E. coli-infected quarters increased much faster than blood PMN CL after both PMA and latex stimulation. Because the luminol-dependent CL kinetics reveal some details about intracellular ROS (DeChatelet et al., 1982; Mehrzad et al., 2001b) and PMN cytoskeleton activity (Sandgren et al., 1992; Meconi et al., 2001), it is reasonable to extrapolate that antimicrobial activity of milk PMN could be further enhanced at PIH 6, 12, and 24. Moreover, the shape of latex-stimulated CL of PMN in $E$. coli infected quarters during the early phase of infection emphasized increased factors related to phagolysosome formation. This could boost $E$. coli exposure to intracellular ROS. The phagolysosome of PMN uses its intracellular myeloperoxidase- $\mathrm{H}_{2} \mathrm{O}_{2}-\mathrm{Cl}$ system to chlorinate bacterial proteins (Rosen et al., 2002), thereby destroying phagocytosed E. coli. Our study showed that peak milk PMN phagocytosis capacity occurred in the early hours of infection. This boosted $E$. coli clearance at d 1 of infection (see Figures 1b, 2, and 5). The weak inten- sity and duration of ROS production of PMN from blood and noninfected quarters indicated that primed blood PMN might have migrated into noninfected quarters. This further confirmed that inflammatory mediators were systemically released.

The particular CL shape reveals the quality of milk PMN. Because oxidative and nonoxidative killing of $E$. coli are interrelated (Belaaouaj et al., 2000; Reeves et al., 2002; Weinrauch et al., 2002), the increased nonoxidative pathway (e.g., elastase) during mastitis (Long et al., 2001; Prin-Mathieu et al., 2002; Moussaoui et al., 2003) boosts the influx of a large amount of ROS into the phagolysosome (Reeves et al., 2002) for $E$. coli destruction. Therefore, the quality and viability of PMN in milk is essential for bacterial removal.

The much higher values of nonstimulated or resting PMN CL in infected quarters might be due to increased production of host-derived cytokines or $E$. coli endotoxin in the infected quarters (Hoeben et al., 2000; Hagiwara et al., 2001), thus increasing milk PMN ROS production capacity and viability. Consistent with previous findings (Hotta et al., 2001; Mehrzad et al., 2001a), PMN ROS capacity in inflamed tissue was higher than in blood. The viability of PMN in the infected quarters 
was similar to that of blood during the early phase of infection. Thus, the number of PMN and SCC, resting PMN CL, and viability of PMN in the quarters during preinfection and early phase of infection may already have started confronting the $E$. coli. This timely physiological reaction of the udder prevented the pathological consequences of mastitis.

Polymorphonuclear neutrophils are both activated and activating cells; and they are the most susceptible cells in milk compartment (Paape et al., 2002; Burvenich et al., 2003). In accordance with Long et al. 2001, MP loss and tissue damage in the infected gland occurred during the day after $E$. coli infection, which can be due to activation of proteolytic enzymes such as elastase, gelatinase, and matrix metalloproteinases. Theoretically, a low viability of resident milk PMN might compromise the total phagocytic and bacteriostatic capacity of the teat cistern (Burvenich et al., 2003), thus facilitating $E$. coli growth, especially during the lag phase of the growth. Nevertheless, it is far more efficient to clear $E$. coli from the teat cistern with newly attracted viable and young PMN capable of effectively engulfing and killing $E$. coli. Indeed, as observed in our study, higher preinfection milk PMN viability enhanced the static phase of the mammary gland's innate defense.

Studies reveal that milk samples of a cow with severe coliform mastitis sometimes do not yield bacterial growth but contain a large number of extracellular ROS, autolytic enzymes, and necrotic PMN that cause mammary tissue damage. The necrotic PMN, as opposed to PMN viability, antagonizes the resolution of inflammation because they are hardly removed by phagocytes and their chromatin proteins boost prolonged inflammation (Scaffidi et al., 2002). Thus, the issue in $E$. coli mastitis is not just the $E$. coli itself but also the resolution of the inflammation (Burvenich et al., 2003). In our opinion, prevention of milk PMN necrosis during early lactation could diminish the severity of $E$. coli mastitis in dairy cows. Although in an early stage of mastitis, this prevention seems unnecessary because the milk PMN viability is physiologically increased (see e.g., Table 2), in severe mastitis cases the increased milk PMN viability is delayed. In our study, mammary tissue damage and MP loss would have been more pronounced if milk PMN viability had not increased during the early hours of mastitis. The current study demonstrated that improving milk PMN viability immediately before infection, at the early hours of infection, and during the resolution of mastitis would prevent extensive mammary tissue damage, thereby shortening mastitis recovery time.

The modulation of bovine PMN viability in milk by $E$. coli mastitis is related to the kinetics of PMN diapedesis through the bone marrow-blood barrier and blood-milk barrier. One of the mechanisms of increased milk PMN viability during mastitis can be the antiapoptotic effect of cytokines, for example, tumor necrosis factor- $\alpha$, interleukin-1, and interleukin-6 (Colotta et al., 1992; Lee et al., 1993; Sweeney et al., 1998; Boulanger et al., 2001) or expression of antiapoptotic and proliferative genes (Long et al., 2001). Milk concentrations of these cytokines and gene expressions are extremely high throughout the d 1 of $E$. coli and endotoxin mastitis (Shuster et al., 1993; Hoeben et al., 2000; Hagiwara et al., 2001). nuclear factor $\kappa \mathrm{B}$ is activated in PMN in the inflamed tissue (Hotta et al., 2001), and its concentration increases in infected quarters (Boulanger et al., 2003). This may contribute to the increased milk PMN viability. Furthermore, the continuous production of apoptosis-inhibiting protein(s) and incomplete activation of caspase-3 (Hotta et al., 2001) and hypoxia-inducible transcription factor-1 (HIF-1) alpha in the site of inflammation (Cramer et al., 2003) prevent cytokineinduced apoptosis and increase the PMN cytosolic ATP pool. This could boost PMN viability in the infected quarters. The contribution of NADPH-oxidase, as its activity increases during mastitis (Mehrzad et al., 2001a), to PMN survival and cytosolic $\mathrm{pH}$ homeostasis is also pivotal (Mayer et al., 1989; Jankowski et al., 2002); the NADPH-oxidase activity boosts ROS production and exerts a $\mathrm{pH}$-buffering effect. This potentially antagonizes the PMN cytosolic acidosis and subsequent necrosis.

The direct action of $E$. coli endotoxin also boosts PMN survival in milk (Boulanger et al., 2001). Furthermore, the fast diapedesis may explain the delayed apoptosis and increased viability of PMN at the site of infection (Ferrante, 1992; Lee et al., 1993; Mehrzad et al., 2001a). The contribution of the blood-milk barrier (Van Oostveldt et al., 2002) and mammary gland injury (Sladek and Rysanek, 2001) in the modulation of PMN apoptosis and viability has been demonstrated. The appearance of metamyelocytes and band cells in milk also contributes to increased milk PMN viability. In our study, the maximal appearance of macrophages at PIH 48 and 72 could also be a contributor to the termination of mastitis.

Overall, during the early phase of mastitis, blood PMN is not activated substantially; however, when PMN migrate in infected quarters their functionalities, viability and CL activity increase sharply. This results mainly from the local effect of the $E$. coli mastitis. The present study indicates that PMN viability and CL in milk are inextricably interrelated, in healthy and in $E$. coli mastitic cows. As a whole, our findings strongly suggest that low resident milk PMN viability could be considered as a risk factor for severe coliform mastitis. This supports the idea of boosting milk PMN viability 
as an appropriate strategy for mastitis prevention and treatment.

\section{ACKNOWLEDGMENTS}

This work was supported in part by the Flemish Institute for the Encouragement of Research in the Industry (IWT grant no. 030784), and the Ministry of Science, Research, and Technology of Iran (stipendium J. Mehrzad). We also thank K. Demeyere and E. Vander Elstraeten for technical assistance.

\section{REFERENCES}

Allen, R. C., R. L. Stjernholm, and R. H. Steele. 1972. Evidence for the generation of an electronic excitation state(s) in human polymorphonuclear leukocytes and its participation in bactericidal activity. Biochem. Biophys. Res. Commun. 47:679-684.

Belaaouaj, A., K. Kim, and S. Shapiro. 2000. Degradation of outer membrane protein A in Escherichia coli killing by neutrophil elastase. Science 289:1185-1188.

Boulanger, V., L. Bouchard, X. Zhao, and P. Lacasse. 2001. Induction of nitric oxide production by bovine mammary epithelial cells and blood leukocytes. J. Dairy Sci. 84:1430-1437.

Boulanger, D., F. Bureau, D. Melotte, J. Mainil, and P. Lekeux. 2003. Increased nuclear factor kappaB activity in milk cells of mastitisaffected cows. J. Dairy Sci. 86:1259-1267.

Burvenich, C., V. Van Merris, J. Mehrzad, A. Diez-Fraile, and L. Duchateau. 2003. Severity of E. coli mastitis is mainly determined by cow factors. Vet. Res. 34:521-562.

Colotta, F., F. Re, N. Polentarutti, S. Suzzani, and A. Mantovani. 1992. Modulation of granulocyte survival and programmed cell death by cytokines and bacterial products. Blood 80:2012-2020.

Cramer, T., Y. Yamanishi, B. E. Clausen, I. Forster, R. Pawlinski, N. Mackman, V. H. Haase, R. Jaenisch, M. Corr, V. Nizet, G. S. Firestein, H. P. Gerber, N. Ferrara, and R. S. Johnson. 2003. HIF-1 alpha is essential for myeloid cell-mediated inflammation. Cell 112:645-657.

DeChatelet, L. R., G. D. Long, P. S. Shirley, D. A. Bass, M. J. Thomas, F. W. Henderson, and M. S. Cohen. 1982. Mechanism of the luminol-dependent chemiluminescence of human neutrophils. J. Immunol. 129:1589-1593.

Ferrante, A. 1992. Activation of neutrophils by interleukins-1 and 2 and tumor necrosis factors. Immunol. Ser. 57:417-436.

Goldberg, J. J., J. W. Pankey, I. Politis, B. Zavizion, and A. J. Bramley. 1995. Effect of oxygen tension on killing of Escherichia coli by bovine polymorphonuclear neutrophil leucocytes in vitro. J. Dairy Res. 62:331-338.

Grebner, J. V., E. L. Mills, B. H. Gray, and P. G. Quie. 1977. Comparison of phagocytic and chemiluminescence response of human polymorphonuclear neutrophils. J. Lab. Clin. Med. 89:153-159.

Griffin, F. M. J., J. A. Griffin, J. E. Leider, and S. C. Silverstein. 1975. Studies on the mechanism of phagocytosis. I. Requirements for circumferential attachment of particle-bound ligands to specific receptors on the macrophage plasma membrane. J. Exp. Med. 142:1263-1282.

Hagiwara, K., H. Yamanaka, K. Hisaeda, S. Taharaguchi, R. Kirisawa, and H. Iwai. 2001. Concentration of IL-6 in serum and whey from healthy and mastitic cows. Vet. Res. Commun. 25:99-108.

Heyneman, R., C. Burvenich, and R. Vercauteren. 1990. Interaction between the respiratory burst activity of neutrophil leukocytes and experimentally induced $E$. coli mastitis in cows. J. Dairy Sci. 73:985-994.

Hill, A. W., A. L. Shears, and K. G. Hibbitt. 1979. The pathogenesis of experimental Escherichia coli mastitis in newly calved dairy cows. Res. Vet. Sci. 26:97-101.

Hoeben, D., C. Burvenich, A. M. Massart-Leën, M. Lenjou, G. Nijs, D. Van Bockstaele, and J. F. Beckers. 1999. In vitro effect of ketone bodies, glucocorticosteroids and bovine pregnancy-associated glycoprotein on cultures of bone marrow progenitor cells of cows and calves. Vet. Immunol. Immunopathol. 68:229-240.

Hoeben, D., C. Burvenich, E. Trevisi, G. Bertoni, J. Hamann, and J. W. Blum. 2000. Role of endotoxin and TNF- $\alpha$ in the pathogenesis of experimentally induced mastitis in periparturient cows. J. Dairy Res. 67:503-514.

Hotta, K., M. Niwa, A. Hara, T. Ohno, X. Wang, H. Matsuno, O. Kozawa, H. Ito, K. Kato, T. Otsuka, N. Matsui, and T. Uematsu. 2001. The loss of susceptibility to apoptosis in exudated tissue neutrophils is associated with their nuclear factor-kappa B activation. Eur. J. Pharmacol. 433:17-27.

Jankowski, A., C. C. Scott, and S. Grinstein. 2002. Determinants of phagosomal pH in neutrophils. J. Biol. Chem. 277:6059-6066.

Kehrli, M. E., Jr., and D. E. Shuster. 1994. Factors affecting milk somatic cells and their role in health of the bovine mammary gland. J. Dairy Sci. 77:619-675.

Lee, A., M. K. B. Whyte, and C. Haslett. 1993. Inhibition of apoptosis and prolongation of neutrophil functional longevity by inflammatory mediators. J. Leukoc. Biol. 54:283-288.

Long, E., A. V. Capuco, D. L. Wood, T. Sonstegard, G. Tomita, M. J. Paape, and X. Zhao. 2001. Escherichia coli induces apoptosis and proliferation of mammary cells. Cell Death Differ. 8:808-816.

Mayer, S. J., P. M. Keen, N. Craven, and F. J. Bourn. 1989. Regulation of phagolysosome $\mathrm{pH}$ in bovine and human neutrophils: The role of NADPH oxidase activity and an $\mathrm{Na}+/ \mathrm{H}+$ antiporter. J. Leukoc. Biol. 45:239-248.

Meconi, S., C. Capo, M. Remacle-Bonnet, G. Pommier, D. Raoult, and J. L. Mege. 2001. Activation of protein tyrosine kinases by Coxiella burnetii: Role in actin cytoskeleton reorganization and bacterial phagocytosis. Infect. Immun. 69:2520-2526.

Mehrzad, J., H. Dosogne, E. Meyer, and C. Burvenich. 2001a. Local and systemic effects of endotoxin mastitis on the chemiluminescence of milk and blood neutrophils in dairy cows. Vet. Res. 32:131-144.

Mehrzad, J., H. Dosogne, E. Meyer, R. Heyneman, and C. Burvenich. 2001b. Respiratory burst activity of blood and milk neutrophils in dairy cows during different stages of lactation. J. Dairy Res. 68:399-415.

Mehrzad, J., H. Dosogne, F. Vangroenweghe, and C. Burvenich. 2001c. A comparative study of bovine blood and milk neutrophil functions with luminol-dependent chemiluminescence. Luminescence 16:343-356.

Mehrzad, J., L. Duchateau, S. Pyörälä, and C. Burvenich. 2002. Blood and milk neutrophil chemiluminescence and viability in primiparous and pluriparous dairy cows during late pregnancy, around parturition and early lactation. J. Dairy Sci. 85:3268-3276.

Moussaoui, F., F. Laurent, J. M. Girardet, G. Humbert, J. L. Gaillard, and Y. Le Roux. 2003. Characterization and proteolytic origins of specific peptides appearing during lipopolysaccharide experimental mastitis. J. Dairy Sci. 86:1163-1170.

Naidu, T. G., and F. H. Newbould. 1973. Glycogen in leukocytes from bovine blood and milk. Can. J. Comp. Med. Vet. Sci. 37:47-55.

Paape, M. J., D. D. Bannerman, X. Zhao, and J. W. Lee. 2003. The bovine neutrophil: Structure and function in blood and milk. Vet. Res. 34:597-627.

Paape, M., J. Mehrzad, X. Zhao, J. Detilleux, and C. Burvenich. 2002. Defense of the bovine mammary gland by polymorphonuclear neutrophil leukocytes. J. Mammary Gland Biol. Neoplasia 7:109-121.

Piccinini, R., V. Bronzo, P. Moroni, P. Luzzago, and A. Zecconi. 1999. Study on the relationship between milk immune factors and Staphylococcus aureus intramammary infections in dairy cows. J. Dairy Res. 66:501-510.

Prin-Mathieu, C., Y. Le Roux, G. C. Faure, F. Laurent, M. C. Bene, and F. Moussaoui. 2002. Enzymatic activities of bovine peripheral blood leukocytes and milk polymorphonuclear neutrophils during intramammary inflammation caused by lipopolysaccharide. Clin. Diagn. Lab. Immunol. 9:812-817.

Reeves, E. P., H. Lu, H. L. Jacobs, C. G. M. Messina, S. Bolsover, G. Gabella, E. O. Potma, A. Warley, J. Roes, and A. W. Segal. 2002. 
Killing activity of neutrophils is mediated through activation of proteases by K+ flux. Nature 416:291-297.

Rosen, H., J. R. Crowley, and J. W. Heinecke. 2002. Human neutrophils use the myeloperoxidase-hydrogen peroxide-chloride system to chlorinate but not nitrate bacterial proteins during phagocytosis. J. Biol. Chem. 277:30463-30468.

Sandgren, C. H., I. Larsson, and K. Persson. 1992. Bovine neutrophils recruited by endotoxin to a teat cistern continuously produce oxygen radicals and show increased phagocytosis and extracellular chemiluminescence. Inflammation 16:117-133.

Scaffidi, P., T. Misteli, and M. E. Bianchi. 2002. Release of chromatin protein HMGB1 by necrotic cells triggers inflammation. Nature 418:191-195.

Shuster, D. E., M. E. Kehrli, and M. G. Stevens. 1993. Cytokine production during endotoxin-induced mastitis in lactating dairy cows. Am. J. Vet. Res. 54:80-85.

Sladek, Z., and D. Rysanek. 2001. Neutrophil apoptosis during the resolution of bovine mammary gland injury. Res. Vet. Sci. $70: 41-46$.
Sweeney, J. F., P. K. Nguyen, G. M. Omann, and D. B. Hinshaw. 1998. Lipopolysaccharide protects polymorphonuclear leukocytes from apoptosis via tyrosine phosphorylation-dependent signal transduction pathways. J. Surg. Res. 74:64-70.

Van Merris, V., E. Meyer, and C. Burvenich. 2002. Functional maturation during bovine granulopoiesis. J. Dairy Sci. 85:2859-2868.

Van Oostveldt, K., M. J. Paape, and C. Burvenich. 2002. Apoptosis of bovine neutrophils following diapedesis through a monolayer of endothelial and mammary epithelial cells. J. Dairy Sci. 85:139-147.

Vandeputte-Van Messom, G., C. Burvenich, E. Roets, A. M. MassartLeën, R. Heyneman, W. D. J. Kremer, and A. Brand. 1993. Classification of newly calved cows into moderate and severe responders to experimentally induced Escherichia coli mastitis. J. Dairy Res. 60:19-29.

Weinrauch, Y., D. Drujan, S. Shapiro, J. Weiss, and A. Zychlinsky. 2002. Neutrophil elastase targets virulence factors of enterobacteria. Nature 417:91-94. 